\title{
A Novel Report on the Compassionate Use of Baricitinib in Treating a Pediatric Patient With Severe Symptoms of COVID-19 Infection
}

\author{
Francis Atemnkeng, ${ }^{\mathrm{a}, \mathrm{b}}$, Harith Alataby ${ }^{\mathrm{a}}$, Jack Demirjian ${ }^{\mathrm{a}}$, Foma Munoh Kenne ${ }^{\mathrm{a}}$, Jay Nfonoyim ${ }^{\mathrm{a}}$
}

\begin{abstract}
Since the outbreak of the pandemic coronavirus disease 2019 (COVID-19), there has been an increasing need for treatment to decrease morbidity and mortality of patients presenting with severe disease symptoms. There has been increasing evidence to suggest that the pathophysiological basis is a severe inflammatory response that resembles the cytokine release syndrome. Current strategies to counteract this involve modifiers of the immune response such as interleukin (IL)-6 receptor blockers and Janus kinase (JAK) inhibitors. An example of a JAK inhibitor is baricitinib. In this case, we present a 17-yearold female admitted with severe COVID-19 symptoms, who was placed on high-flow nasal cannula and started on azithromycin and hydroxychloroquine, which were standard of care at the time. Due to the worsening of symptoms, she was given baricitinib for compassionate use. There was a rapid improvement in clinical and imaging findings, and the patient was discharged from the hospital within 8 days of admission. This study is fascinating because there are very limited studies published on the benefits of baricitinib in managing patients with severe symptoms of COVID-19 especially in the pediatric population, and the rapidity in recovery time was remarkable.
\end{abstract}

Keywords: COVID-19; Baricitinib; Cytokine; Inflammation

\section{Introduction}

Ever since the first case of the novel coronavirus disease 2019 (COVID-19) was identified, over 63 million new cases have been reported, with close to 1.5 million deaths worldwide. The active viral phase consists of influenza-like symptoms after incubation, which might progress to severe symptoms, including hypoxemic respiratory failure $[1,2]$. Several mechanisms have

Manuscript submitted December 1, 2020, accepted December 21, 2020 Published online January 19, 2021

aRichmond University Medical Center, Staten Island, NY, USA

${ }^{\mathrm{b}}$ Corresponding Author: Francis Atemnkeng, Richmond University Medical Center, Staten Island, New York, USA. Email: fatemnkeng@rumcsi.org

doi: https://doi.org/10.14740/jmc3629 been explained in various ways through which the COVID-19 can cause symptoms in humans. One of these mechanisms is an exaggerated inflammatory response to the virus and the development of cytokine storm implicated in respiratory failure and multiorgan damage $[3,4]$. Several treatment options have been explored, especially for hospitalized patients, to counteract the disease process. Examples of treatment options include antimalarial drugs [5], antiviral agents [6], immunomodulators [7-12], glucocorticoids $[13,14]$ and convalescent plasma [15, $16]$ with very varying results. Baricitinib is an immunomodulator that has shown some promise in managing COVID-19 patients with severe symptoms [8, 9]. It inhibits the Janus kinase (JAK) proteins, which are greatly involved in the hyperinflammatory response and cytokine storm formation [3, 4]. In this article, we present a case of severe COVID-19 infection complicated by acute hypoxic respiratory failure who was successfully treated with baricitinib, in addition to hydroxychloroquine and azithromycin. At the time of the presentation, remdesivir had only emergency use authorization, so it was provided only by the Department of Health for the state, with a very limited supply available at the time.

\section{Case Report}

A 17-year-old obese female with a nonsignificant past medical history presented to the emergency room (ER) because of fever, cough, and shortness of breath. Onset 7 days before admission with ungraded fever and cough, was aggravated by dyspnea, which prompted her to call the emergency medical services (EMS). The EMS reported that the patient's oxygen saturation was in the low $80 \mathrm{~s}$, so she was placed on a nonrebreather mask (NRM) and transferred to the hospital. Upon arrival in the ER, the patient looked lethargic, in severe respiratory distress, and could only speak two to three words per sentence. On arrival, vitals were as follows: blood pressure (BP) $98 / 69$, pulse 140 , respiratory rate 30 , saturation $98 \%$ on $\mathrm{NRM}$, and temperature of $38.4{ }^{\circ} \mathrm{C}$. On physical examination, there were fine crepitations in both lung fields. Real-time polymerase chain reaction (PCR) for COVID-19 was performed, which came back positive. Chest X-ray showed patchy bilateral opacities (Fig. 1). Other laboratory studies revealed white blood cell (WBC): $13.7 \times 10^{3} / \mu \mathrm{L}$, hemoglobin $(\mathrm{Hb}): 12.5 \mathrm{~g} /$ $\mathrm{dL}$, erythrocyte sedimentation rate (ESR): $95 \mathrm{~mm} / \mathrm{h}, \mathrm{D}$-dimer: 

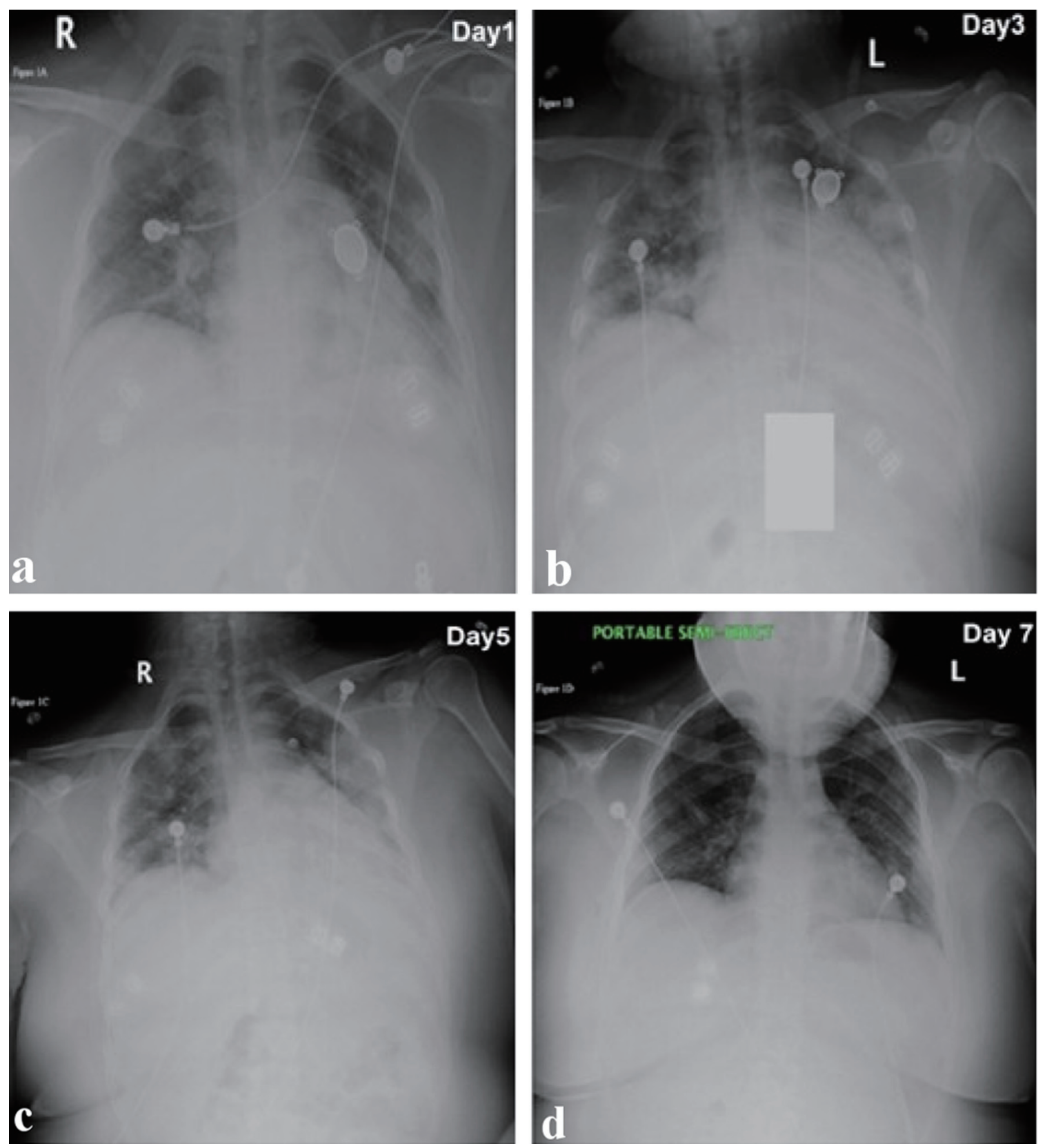

Figure 1. Series of chest X-ray images from the day of admission to the last day of admission. (a) Retrocardiac consolidation and patchy infiltrate in the left upper to mid lung fields and patchy infiltrate in the right lung, suspicious for pneumonic infiltrate (day 1). (b) There are bilateral patchy infiltrates unchanged, with no pleural fluid, and no pneumothorax (day 3). (c) Markedly suboptimal depth of inspiration. Bilateral hazy to patchy pneumonic infiltrates persist, but mild improvement from previous study (day 5). (d) Shallow depth of inspiration, mild atelectasis in the right perihilar distribution, and lungs otherwise grossly clear (day 7).

$0.72 \mu \mathrm{g} / \mathrm{mL}$, C-reactive protein (CRP): $17.1 \mathrm{mg} / \mathrm{dL}$, ferritin: $251.2 \mathrm{ng} / \mathrm{mL}$, blood urea nitrogen (BUN): $6 \mathrm{mg} / \mathrm{dL}$ and creatinine: $0.5 \mathrm{mg} / \mathrm{dL}$. The patient was admitted to the pediatric intensive care unit (ICU); the NRM was switched to high-flow nasal cannula (HFNC) (45 L per minute (LPM) at $50 \% \mathrm{FiO}_{2}$ ) and was started on hydroxychloroquine, azithromycin, vitamin $\mathrm{C}$, and zinc, which were standard of care at the time. Remdesivir was requested from the Department of Health but was not readily available due to limited supply. On day 2 of admis- sion, her condition worsened, with a respiratory rate in the 40 $\mathrm{s}$, severe respiratory distress with the use of accessory muscles. She was then placed on a non-invasive positive pressure ventilation machine on continuous positive airway pressure (CPAP) mode to improve the oxygenation. The chest X-ray also showed worsening in her lung infiltrates (Fig. 1). At this point, she had a very low threshold for intubation due to her worsening clinical state. This prompted the medical team to discuss other options available to manage the patient, and it 
Table 1. Markers of Inflammation Measured Daily From Admission to Discharge

\begin{tabular}{lllllll} 
Day & $\mathbf{1}$ & $\mathbf{2}$ & $\mathbf{3}$ & $\mathbf{4}$ & $\mathbf{5}$ & $\mathbf{6}$ \\
\hline CRP $(\mathrm{mg} / \mathrm{mL})$ & 14.1 & 17.1 & 7.92 & 3.46 & 1.29 & 0.638 \\
ESR $(\mathrm{mm} / \mathrm{h})$ & 67 & 126 & 96 & 95 & - & 55 \\
Ferritin $(\mathrm{ng} / \mathrm{mL})$ & - & 251.2 & 239.3 & 215.2 & 179.7 & - \\
\hline
\end{tabular}

CRP: C-reactive protein; ESR: erythrocyte sedimentation rate.

was finally decided to start on immunomodulators for compassionate use. So the patient was started on baricitinib $4 \mathrm{mg}$ oral dose, given daily for 5 days.

On day 3, there was a marked improvement in the patient's clinical status, no longer in respiratory distress, with a respiratory rate 18 - 22; she looked comfortable, not using any accessory muscles. Vitals showed BP 116/55 mm Hg, heart rate (HR) 97, temperature of $37.1^{\circ} \mathrm{C}$. Oxygen delivery mode was deescalated from CPAP back to HFNC $50 \mathrm{LPM}$ and $50 \% \mathrm{FiO}_{2}$, which was further deescalated to Venturi mask, and then nasal cannula. Repeat chest X-ray showed a marked improvement in the opacities.

By day 6 of admission, the patient was comfortable with ambient air, saturating $98 \%$. The patchy opacities on the chest $\mathrm{X}$-ray was resolving significantly; and the inflammatory markers are improved (Table 1). She was discharged home on day 8 and scheduled for follow-up in the pediatric clinic.

\section{Discussion}

Ever since discovering the first case of COVID-19, there have been over 63 million cases reported worldwide, and the number of deaths averaged over 1.4 million. Most patients present with either mild non-specific symptoms or present with severe pneumonia with multiorgan dysfunction [17]. Various patho- physiologic processes have been suggested, one of which is an exaggerated inflammatory response to the virus and the development of cytokine storm implicated in respiratory failure and multiorgan damage $[3,4]$. Patients will tend to have very high inflammatory markers, including serum interleukin (IL)6 , ferritin, and CRP levels. Our patient came to the hospital with respiratory distress with increased oxygen requirements. This could be explained by the elevated inflammatory markers (Table 1), which she presented at admission. This might have progressed to cytokine storm, mediated by IL-6, which could explain her worsening clinical and radiographic findings.

When IL- 6 binds to its receptors on the membrane of inflammatory cells, it may stimulate the activation of these cells via the Janus kinase/signal transducer and activator transcription (JAK/STAT) pathway, as well as the nuclear factor kappa $\mathrm{B} /$ mitogen-activated protein kinase (NF-KB/MAPK) pathway leading to exaggerated inflammatory response and massive cytokine storm [18]. Baricitinib inhibits the JAK/STAT pathway; this inhibition causes the lowering of IL-6 levels and reduces the cytokine storm (Fig. 2). There has been increasing evidence of the efficacy of baricitinib in treating COVID-19 patients, decreasing the cytokine storm, and decreasing the morbidity, mortality, and length of stay in the hospital [12]. Our patient received a 5-day course of baricitinib; and after the first dose, there was a remarkable improvement in her clinical state with decreasing oxygen requirements. There was also a rapid

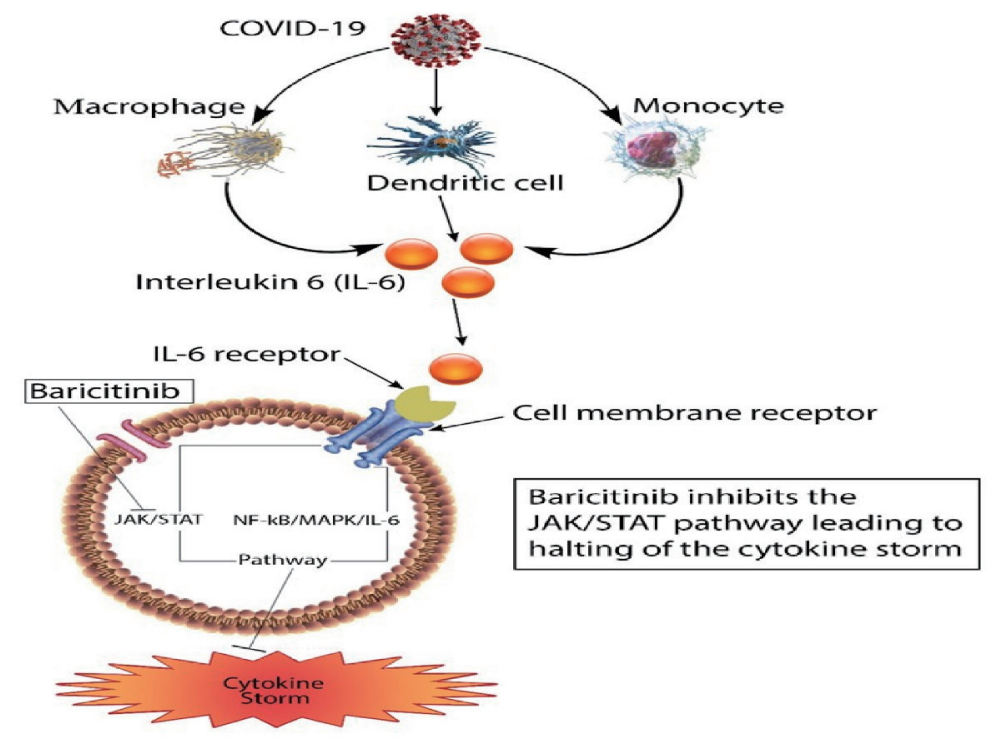

Figure 2. Schematic diagram showing the inhibition of JAK/STAT by baricitinib. JAK/STAT: Janus kinase/signal transducer and activator transcription; COVID-19: coronavirus disease 2019. 
clearing of the opacities in the chest X-rays upon completion of baricitinib. Even though this could be the natural history of the disease, the acuity of the improvement after starting baricitinib warrants more research on the benefits of this medication in treating COVID-19 patients with severe symptoms and elevated inflammatory markers.

This case is exciting because there are very rare case reports on the benefits of baricitinib on the treatment of COVID-19 patients, especially in the pediatric population where there are rare cases of severe symptoms. This could be useful for more research on its use on infants and adolescents who present with severe symptoms of COVID-19 as more research needs to be conducted to assess the safety and efficacy.

\section{Conclusions}

The use of baricitinib in the treatment of COVID-19 has shown some promise in decreasing morbidity, mortality, and length of stay in the hospital. There are very limited reports on its use in the pediatric population; and our case shown here might have some benefits in initiating this medication in this group. Further research is needed to determine these benefits.

\section{Acknowledgments}

None to declare.

\section{Financial Disclosure}

None to declare.

\section{Conflict of Interest}

The authors report no conflict of interest.

\section{Informed Consent}

Not applicable.

\section{Author Contributions}

F. Atemnkeng collected the data, guided the literature search, wrote the manuscript, and is the research guarantor. H. Alataby, J. Demirjian, and F. Kenne helped with data collection and writing of the article. J. Nfonoyim reviewed and supervised the study.

\section{Data Availability}

The authors declare that data supporting the findings of this study are available within the article.

\section{References}

1. Guan WJ, Ni ZY, Hu Y, Liang WH, Ou CQ, He JX, Liu L, et al. Clinical characteristics of coronavirus disease 2019 in China. N Engl J Med. 2020;382(18):1708-1720.

2. Gandhi RT, Lynch JB, Del Rio C. Mild or moderate COVID-19. N Engl J Med. 2020;383(18):1757-1766.

3. Guo T, Fan Y, Chen M, Wu X, Zhang L, He T, Wang H, et al. Cardiovascular implications of fatal outcomes of patients with coronavirus disease 2019 (COVID-19). JAMA Cardiol. 2020;5(7):811-818.

4. Shi S, Qin M, Shen B, Cai Y, Liu T, Yang F, Gong W, et al. Association of cardiac injury with mortality in hospitalized patients with COVID-19 in Wuhan, China. JAMA Cardiol. 2020;5(7):802-810.

5. Cavalcanti AB, Zampieri FG, Rosa RG, Azevedo LCP, Veiga VC, Avezum A, Damiani LP, et al. Hydroxychloroquine with or without Azithromycin in Mild-to-Moderate Covid-19. N Engl J Med. 2020;383(21):2041-2052.

6. Beigel JH, Tomashek KM, Dodd LE, Mehta AK, Zingman BS, Kalil AC, Hohmann E, et al. Remdesivir for the treatment of COVID-19 - final report. N Engl J Med. 2020;383(19):1813-1826.

7. Sims JT, Krishnan V, Chang CY, Engle SM, Casalini G, Rodgers GH, Bivi N, et al. Characterization of the cytokine storm reflects hyperinflammatory endothelial dysfunction in COVID-19. J Allergy Clin Immunol. 2020.

8. Bronte V, Ugel S, Tinazzi E, Vella A, De Sanctis F, Cane $\mathrm{S}$, Batani $\mathrm{V}$, et al. Baricitinib restrains the immune dysregulation in patients with severe COVID-19. J Clin Invest. 2020;130(12):6409-6416.

9. Stebbing J, Krishnan V, de Bono S, Ottaviani S, Casalini G, Richardson PJ, Monteil V, et al. Mechanism of baricitinib supports artificial intelligence-predicted testing in COVID-19 patients. EMBO Mol Med. 2020;12(8): e12697.

10. Dastan F, Saffaei A, Haseli S, Marjani M, Moniri A, Abtahian Z, Abedini A, et al. Promising effects of tocilizumab in COVID-19: A non-controlled, prospective clinical trial. Int Immunopharmacol. 2020;88:106869.

11. Stone JH, Frigault MJ, Serling-Boyd NJ, Fernandes AD, Harvey L, Foulkes AS, Horick NK, et al. Efficacy of tocilizumab in patients hospitalized with COVID-19. N Engl J Med. 2020;383(24):2333-2344.

12. Titanji BK, Farley MM, Mehta A, Connor-Schuler R, Moanna A, Cribbs SK, O'Shea J, et al. Use of Baricitinib in patients with moderate and severe COVID-19. Clin Infect Dis. 2020

13. Recovery Collaborative Group, Horby P, Lim WS, Emberson JR, Mafham M, Bell JL, Linsell L, et al. Dexamethasone in hospitalized patients with COVID-19 - preliminary report. N Engl J Med. 2020.

14. W. H. O. Rapid Evidence Appraisal for COVID-19 Therapies Working Group, Sterne JAC, Murthy S, Diaz JV, Slutsky AS, Villar J, Angus DC, et al. Association between administration of systemic corticosteroids and mortality among critically ill patients with COVID-19: A Meta-analysis. JAMA. 2020;324(13):1330-1341. 
15. Li L, Zhang W, Hu Y, Tong X, Zheng S, Yang J, Kong $\mathrm{Y}$, et al. Effect of convalescent plasma therapy on time to clinical improvement in patients with severe and lifethreatening COVID-19: A Randomized Clinical Trial. JAMA. 2020;324(5):460-470.

16. Joyner MJ, Senefeld JW, Klassen SA, Mills JR, Johnson PW, Theel ES, et al. Effect of convalescent plasma on mortality among hospitalized patients with COVID-19: initial three-month experience. medRxiv. 2020.

17. Ge H, Wang X, Yuan X, Xiao G, Wang C, Deng T, Yuan Q, et al. The epidemiology and clinical information about COVID-19. Eur J Clin Microbiol Infect Dis. 2020;39(6):1011-1019.

18. Mahmudpour M, Roozbeh J, Keshavarz M, Farrokhi S, Nabipour I. COVID-19 cytokine storm: the anger of inflammation. Cytokine. 2020;133:155151. 